\section{Effect of Gene Therapy on Visual Function in Leber's Congenital Amaurosis}

\author{
James W.B. Bainbridge, Ph.D., F.R.C.Ophth., Alexander J. Smith, Ph.D., \\ Susie S. Barker, Ph.D., Scott Robbie, M.R.C.Ophth., \\ Robert Henderson, M.R.C.Ophth., Kamaljit Balaggan, M.R.C.Ophth., \\ Ananth Viswanathan, M.D., F.R.C.Ophth., Graham E. Holder, Ph.D., \\ Andrew Stockman, Ph.D., Nick Tyler, Ph.D., Simon Petersen-Jones, Ph.D., \\ Shomi S. Bhattacharya, Ph.D., Adrian J. Thrasher, Ph.D., M.R.C.P., F.R.C.P., \\ Fred W. Fitzke, Ph.D., Barrie J. Carter, Ph.D., Gary S. Rubin, Ph.D., \\ Anthony T. Moore, F.R.C.Ophth., and Robin R. Ali, Ph.D.
}

\section{SUMMARY}

Early-onset, severe retinal dystrophy caused by mutations in the gene encoding retinal pigment epithelium-specific $65-\mathrm{kDa}$ protein (RPE65) is associated with poor vision at birth and complete loss of vision in early adulthood. We administered to three young adult patients subretinal injections of recombinant adeno-associated virus vector 2/2 expressing RPE65 complementary DNA (cDNA) under the control of a human RPE65 promoter. There were no serious adverse events. There was no clinically significant change in visual acuity or in peripheral visual fields on Goldmann perimetry in any of the three patients. We detected no change in retinal responses on electroretinography. One patient had significant improvement in visual function on microperimetry and on dark-adapted perimetry. This patient also showed improvement in a subjective test of visual mobility. These findings provide support for further clinical studies of this experimental approach in other patients with mutant RPE65. (ClinicalTrials.gov number, NCT00643747.)

\footnotetext{
L
}

EBER'S CONGENITAL AMAUROSIS IS A TERM USED TO DESCRIBE A GROUP OF recessively inherited, severe, infantile-onset rod-cone dystrophies. ${ }^{1}$ Mutation of one of several genes, including RPE65, causes disease that involves impaired vision from birth ${ }^{2,3}$ and typically progresses to blindness in the third decade of life. There is no effective treatment. RPE65 is expressed in the retinal pigment epitheli$\mathrm{um}$ and encodes a $65-\mathrm{kD}$ protein that is a key component of the visual cycle, ${ }^{1,4-8}$ a biochemical pathway that regenerates the visual pigment after exposure to light. ${ }^{9-14}$ A lack of functional RPE65 results in deficiency of 11-cis retinal so that rod photoreceptor cells are unable to respond to light. Cone photoreceptor cells may have access to 11-cis-retinaldehyde chromophore through an alternative pathway that does not depend on retinal pigment epithelium-derived RPE65,15,16 thus allowing conemediated vision in children with Leber congenital amaurosis. However, progressive degeneration of cone photoreceptor cells ultimately results in loss of cone-mediated vision.

Although the retinal dystrophy caused by defects in RPE65 is severe, features of the disorder suggest that it may respond to gene-replacement therapy. There is use-
From the Institute of Ophthalmology, University College London (J.W.B.B., A.J.S., S.S. Barker, S.R., R.H., K.B., A.V., G.E.H., A.S., S.S. Bhattacharya, F.W.F., G.S.R., A.T.M., R.R.A.); Moorfields Eye Hospital (J.W.B.B., A.V., G.E.H., A.T.M.); the National Institute of Health Research Biomedical Research Centre for Ophthalmology, University College London and Moorfields Eye Hospital (J.W.B.B., G.S.R., A.T.M., R.R.A.); the Department of Civil and Environmental Engineering, University College London (N.T.); and the Institute of Child Health, University College London (A.J.T., R.R.A.) - all in London; Michigan State University, East Lansing (S.P.-J.); and Targeted Genetics Corporation, Seattle (B.J.C.). Address reprint requests to Dr. Ali at the Institute of Ophthalmology, University College London, Bath St., London EClV 9EL, United Kingdom, or at r.ali@ucl.ac.uk.

Drs. Bainbridge and Smith contributed equally to this article.

This article (10.1056/NEJMoa0802268) was published at www.nejm.org on April 27, 2008.

N Engl J Med 2008;358.

Copyright () 2008 Massachusetts Medical Society. 
ful visual function in childhood, and retinal imaging suggests that photoreceptor-cell death occurs late in the disease process. ${ }^{3}$ Gene transfer therefore has the potential to improve visual function as well as preserve existing vision. Gene-replacement therapy has been shown to improve visual function in the Swedish Briard dog, a naturally occurring animal model with mutated RPE65. ${ }^{17}$ Subretinal delivery of recombinant adeno-associated virus vector containing the RPE65 cDNA results in improved retinal function and improved vision, as determined by visual mobility in low light. ${ }^{18-22}$

The purpose of this study was to determine whether gene therapy for retinal dystrophy caused by RPE65 mutations was associated with immediately obvious adverse events and whether efficacy could be demonstrated in humans. In this exploratory, open-label, single-center study involving three young adults, each of whom received a single subretinal injection of recombinant adenoassociated virus 2/2.hRPE65p.hRPE65, the primary outcome was safety, and the secondary outcome was evidence of efficacy in terms of visual function.

METHODS

\section{PATIENTS AND STUDY DESIGN}

In this study, we included young adults (17 to 23 years of age) with early-onset, severe retinal dystrophy caused by missense mutations in RPE65 (Table 1 of the Supplementary Appendix, available with the full text of this article at www.nejm. org). We excluded persons with visual acuity in the study eye that was better than $20 / 120$ on the Snellen visual acuity scale, null mutations, and contraindications to systemic immunosuppression, as well as women who were pregnant or lactating. A National Health Service diagnostic laboratory (Manchester Regional Genetics Laboratory) confirmed the genotypes of potential subjects. In each patient, the eye with the worse acuity was selected as the study eye. The contralateral eye was used as a control.

The study was approved by the U.K. Gene Therapy Advisory Committee, the Medicines and Health Products Regulatory Authority, the Moorfields Research Governance Committee, and the local research ethics committee. All patients gave written informed consent. The study was con- ducted in compliance with Good Clinical Practice guidelines according to the European Clinical Trials Directive (Directive 2001 EU/20/EC) and the Declaration of Helsinki.

Before administration of the vector, we evaluated the retinal structure and function by means of clinical assessment, retinal imaging, psychophysical techniques, and electrodiagnostic methods. Retinal imaging techniques included color fundus photography, fundus autofluorescence imaging, and optical coherence tomography to determine retinal thickness and integrity. We measured visual acuity, contrast sensitivity, color vision, and cone flicker sensitivities. We investigated the patients' visual fields by means of microperimetry (see the Supplementary Appendix), Goldmann dynamic perimetry, and photopic and scotopic (dark-adapted) automated static perimetry (see the Supplementary Appendix). All testing was performed according to standardized, detailed protocols, with controlled room lighting, a dark-adaptation period, and a fixed sequence of test patterns. Both microperimetry and darkadapted perimetry are fully automated, so there was little opportunity for experimenter bias. We determined the visual mobility of the patients at different illumination levels by measuring their ability to navigate a simulated street scene (Fig. 1 in the Supplementary Appendix). Electrophysiological evaluation included full-field, pattern, and multifocal electroretinography performed to incorporate the standards and guidelines of the International Society for Clinical Electrophysiology of Vision.

We repeated assessments of visual function and immune status (see below) at 2, 4, 6, and 12 months (the latter for Patient 1 only; Patients 2 and 3 have not yet reached the 12-month point) after administration of the vector. The end point for toxic effects for each patient was a grade 3 adverse event, defined as loss of visual acuity by 15 or more letters according to the Early Treatment Diabetic Retinopathy Study scale (on which 20/20 denotes perfect vision), or severe, unresponsive intraocular inflammation. The end point for efficacy for each patient was defined as any improvement in visual function that was greater than the test-retest difference for each technique. The assay of immune response and detection of disseminated recombinant adeno-associated virus are described in the Supplementary Appendix. 
RECOMBINANT ADENO-ASSOCIATED VIRUS AND SUBRETINAL DELIVERY

The tgAAG76 vector is a recombinant adenoassociated virus vector of serotype 2 . The vector contains the human RPE65 coding sequence driven by a 1400-bp fragment of the human RPE65 promoter and terminated by the bovine growth hormone polyadenylation site, as described elsewhere. ${ }^{21}$ The vector was produced by Targeted Genetics Corporation according to Good Manufacturing Practice guidelines with the use of a B50 packaging cell line, ${ }^{23}$ an adenovirus-adenoassociated virus hybrid shuttle vector containing the tgAAG76 vector genome, and an adenovirus 5 helper virus. The vector was filled in a buffered saline solution at a titer of $1 \times 10^{11}$ vector particles per milliliter and frozen in 1-ml aliquots at $-70^{\circ} \mathrm{C}$.

Patients 1, 2, and 3 underwent surgery on February 7, April 25, and July 11, 2007, respectively. After three-port vitrectomy, we administered up to $1 \mathrm{ml}$ of recombinant adeno-associated virus vector by means of a subretinal cannula (de Juan, Synergetics) to the subretinal space of one eye, involving up to one third of the total retinal area, including the macula. To reduce the possibility of clinically significant intraocular inflammation, patients were given a 5-week course of oral prednisolone, at a dose of $0.5 \mathrm{mg}$ per kilogram of body weight for 1 week before administration of the vector, $1 \mathrm{mg}$ per kilogram for the first week after administration, $0.5 \mathrm{mg}$ per kilogram for the second week, $0.25 \mathrm{mg}$ per kilogram for the third week, and $0.125 \mathrm{mg}$ per kilogram for the fourth week. Patients received betamethasone and cefuroxime subconjunctivally at the completion of surgery and topical treatment with $0.5 \%$ chloramphenicol four times a day for 7 days, $0.1 \%$ dexamethasone four times a day for 4 weeks, and 1\% atropine twice a day for 7 days after surgery. We performed a clinical examination, fundus photography, and ocular coherence tomography (Stratus OCT, Carl Zeiss Meditec) at frequent intervals in the early postoperative period to monitor for retinal reattachment and to identify any intraocular inflammation.

RESULTS

Each patient had little or no vision in low light from an early age but retained some limited visual function in good lighting conditions. We selected these patients because they retained a limited degree of residual retinal function despite advanced retinal degeneration, and they might therefore be expected to benefit from intervention.

We performed vitrectomy and subretinal injection of the vector without complication in each patient (Fig. 1 and Video 1). The vitreous gel was relatively degenerate; a posterior vitreous detachment was present in Patient 2 and was readily induced in Patients 1 and 3 by active aspiration at the optic disk with the use of the vitreous cutter. To deliver the vector to the relatively wellpreserved retina at the posterior pole, we performed a retinotomy superior to the proximal part of the superotemporal vascular arcade. To minimize injection of the vector into the vitreous or choroid, we first induced a small detachment of the neurosensory retina, using Hartmann's solution before injecting up to $1 \mathrm{ml}$ of recombinant adeno-associated virus vector (thus creating a "bleb") through the same single retinotomy. In Patient 2, the bleb of the vector extended spontaneously across the macula. We actively manipulated the bleb in Patients 1 and $3-$ to involve the macula - by injecting air into the vitreous cavity. We caused no iatrogenic retinal tears, and we left the vector in situ under fluid without retinopexy or intraocular tamponade. On clinical examination 24 hours after surgery, the induced retinal detachment had almost fully resolved in each patient (Fig. 1A). Optical coherence tomography showed minimal persistent subretinal fluid at the macula that resolved 2 to 3 days after surgery (Fig. 1B). On clinical examination, the appearance of the retinas was unchanged for the duration of the follow-up period (up to 12 months).

We detected no dissemination of the vector, as assessed by means of polymerase-chain-reaction amplification of DNA isolated from samples of tears, serum, and saliva collected 1 day and 30 days after administration of the vector and from semen collected at 30 days (data not shown). We observed mild, self-limiting postoperative intraocular inflammation, which typically follows vitrectomy. There were no other adverse events. We found no evidence of cystoid macular edema clinically or on optical coherence tomography. We detected no specific cellular or humoral immune responses to adeno-associated virus capsid (Fig. 2 of the Supplementary Appendix) or specific humoral responses to the transgene prod- 


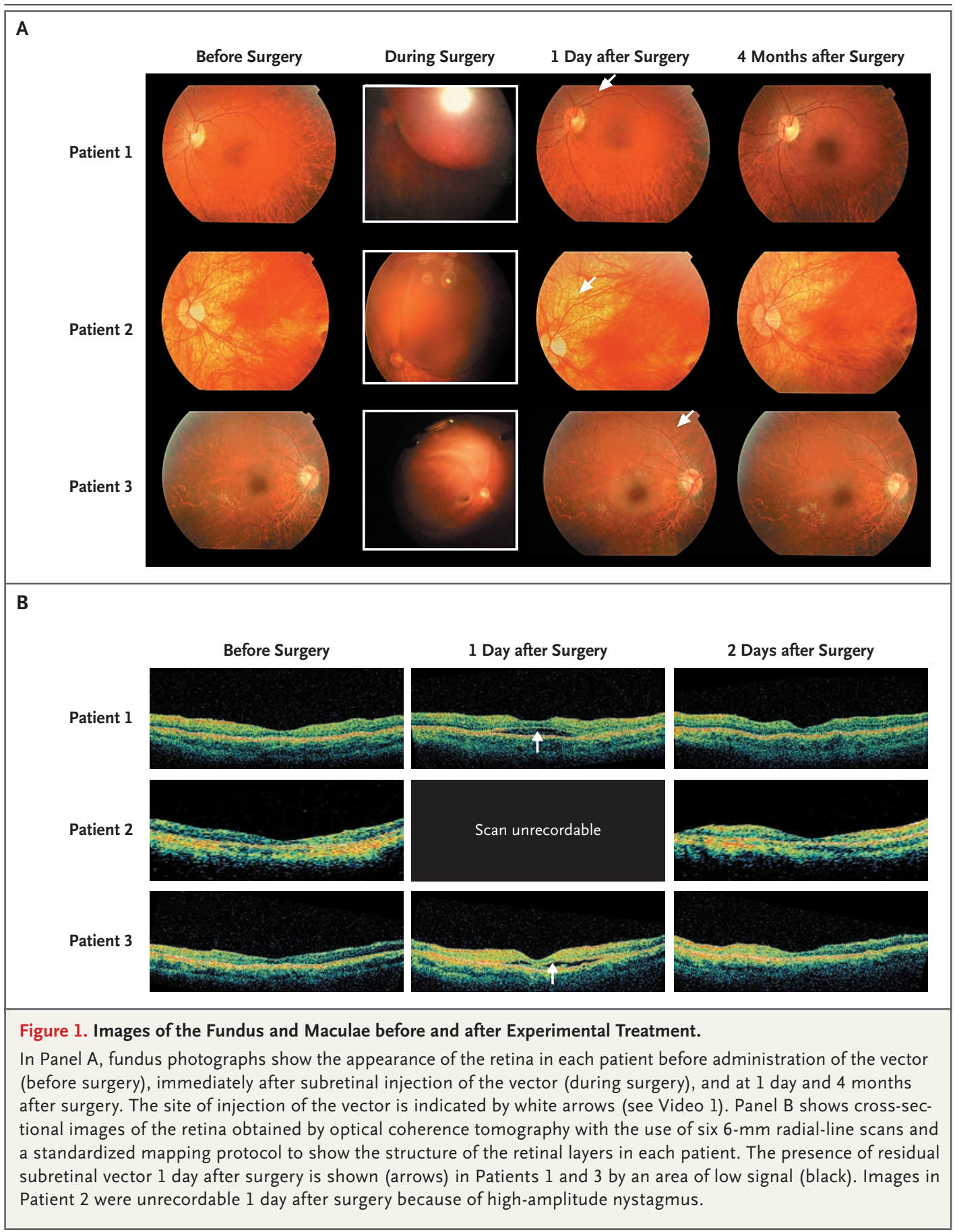

uct (Fig. 2 and Table 2 of the Supplementary Appendix). We detected a small increase in nonspecific activation of $\mathrm{T}$ cells in two patients, which is consistent with a rebound in the numbers of some lymphocyte subgroups after the withdrawal of corticosteroids (Fig. 2A of the Supplementary Appendix).
Visual acuity decreased predictably in association with the temporary retinal detachment induced by administration of the vector and returned to preoperative levels by 6 months (Table 1). We observed no clinically significant improvement in visual acuity in any of the three patients (Table 1) or any change in peripheral visual 


\begin{tabular}{|c|c|c|c|c|c|}
\hline Patient No. and Test & Baseline & $2 \mathrm{Mo}$ & $4 \mathrm{Mo}$ & $6 \mathrm{Mo}$ & $12 \mathrm{Mo}$ \\
\hline \multicolumn{6}{|l|}{1} \\
\hline Study eye — logMAR (Snellen) & $1.16(20 / 286)$ & $1.06(20 / 226)$ & $0.98(20 / 190)$ & & $0.86(20 / 145)$ \\
\hline Control eye — logMAR (Snellen) & $0.88(20 / 150)$ & $0.90(20 / 156)$ & $0.68(20 / 95)$ & & $0.78(20 / 120)$ \\
\hline Study eye $-\log C S$ & 0.05 & 0.30 & 0.60 & & 0.50 \\
\hline Control eye $-\log C S$ & 0.55 & 0.60 & 0.55 & & 0.55 \\
\hline \multicolumn{6}{|l|}{2} \\
\hline Study eye — logMAR (Snellen) & $1.52(20 / 662)$ & $1.50(20 / 632)$ & $1.58(20 / 760)$ & & $1.52(20 / 662)$ \\
\hline Control eye — logMAR (Snellen) & $1.62(20 / 833)$ & $1.56(20 / 662)$ & $1.52(20 / 662)$ & & $1.58(20 / 760)$ \\
\hline Study eye $-\log C S$ & 0.00 & 0.00 & 0.00 & & 0.00 \\
\hline Control eye - logCS & 0.00 & 0.00 & 0.00 & & 0.00 \\
\hline \multicolumn{6}{|l|}{3} \\
\hline Study eye - logMAR (Snellen) & $0.76(20 / 115)$ & $0.90(20 / 156)$ & $0.80(20 / 126)$ & $0.76(20 / 115)$ & \\
\hline Control eye - logMAR (Snellen) & $0.54(20 / 69)$ & $0.46(20 / 58)$ & $0.40(20 / 50)$ & $0.44(20 / 55)$ & \\
\hline Study eye - $\log C S$ & 0.85 & 0.35 & 0.45 & 0.60 & \\
\hline Control eye - logCS & 1.10 & 1.10 & 1.20 & 1.10 & \\
\hline
\end{tabular}

* The logarithm of the minimum angle of resolution $(\log M A R)$ is used here because it allows comparisons of visual acuity scores that are more precise than are scores on the Snellen visual acuity scale. LogCS denotes the logarithm of contrast sensitivity.

fields on Goldmann perimetry testing. We detected no change in retinal responses to flash or pattern electroretinography. Before surgery, Patient 2 had high-amplitude nystagmus, which did not change after treatment.

Microperimetry showed no change in retinal function in Patients 1 and 2 but improved retinal function in Patient 3 (Fig. 2A). The baseline data for Patient 3 were obtained from the average of two measurements taken 1 week apart. Measurements were performed on the same retinal loci by registering the fundus image with the baseline image. In an area extending from the outer macula to a point beyond the major vascular arcade, the retinal sensitivity improved progressively in the right (study) eye by as much as $14 \mathrm{~dB}$ (a factor of 25). Thus, the patient could see small spots of light that were $1 / 25$ th as bright as those that could be seen before treatment. There was no improvement in the left (control) eye.

Dark-adapted perimetry showed no change in retinal function in Patients 1 and 2 but showed improved retinal function in Patient 3 (Fig. 2). In Patients 1 and 2, there was no single location that showed significant improvement or deterioration $(\mathrm{P}<0.05)$. In Patient 3 , some locations in the left (control) eye had yellow bars representing nonsignificant decreases and red bars representing significant decreases in sensitivity. In the right (study) eye, 37 locations showed significant improvements in sensitivity $(\mathrm{P}<0.01)$. The mean sensitivity at nine locations in the inferonasal region improved from $4 \mathrm{~dB}$ at baseline to $26 \mathrm{~dB}$ after treatment, and nine locations in the inferotemporal quadrant improved from $7 \mathrm{~dB}$ to $28 \mathrm{~dB}$. This finding is equivalent to an improvement in sensitivity of more than $20 \mathrm{~dB}$ for these 18 locations, or 100 times the sensitivity threshold observed at baseline.

Visual mobility in low light was unchanged in Patients 1 and 2 but improved in Patient 3 (Fig. 3 and Video 2). In bright conditions, the visually guided mobility in Patient 3 was within normal limits at baseline and follow-up. Under low illumination at baseline, the visual performance of Patient 3 was very poor with the study eye as compared with the control eye (with which he made no errors). At follow-up, we observed a small change for the control eye. We attribute this change to a general learning effect; a similar improvement in travel time to complete the course under dim illumination was also observed in Patient 1. However, after administration of the vector, the travel time for Patient 3 improved 


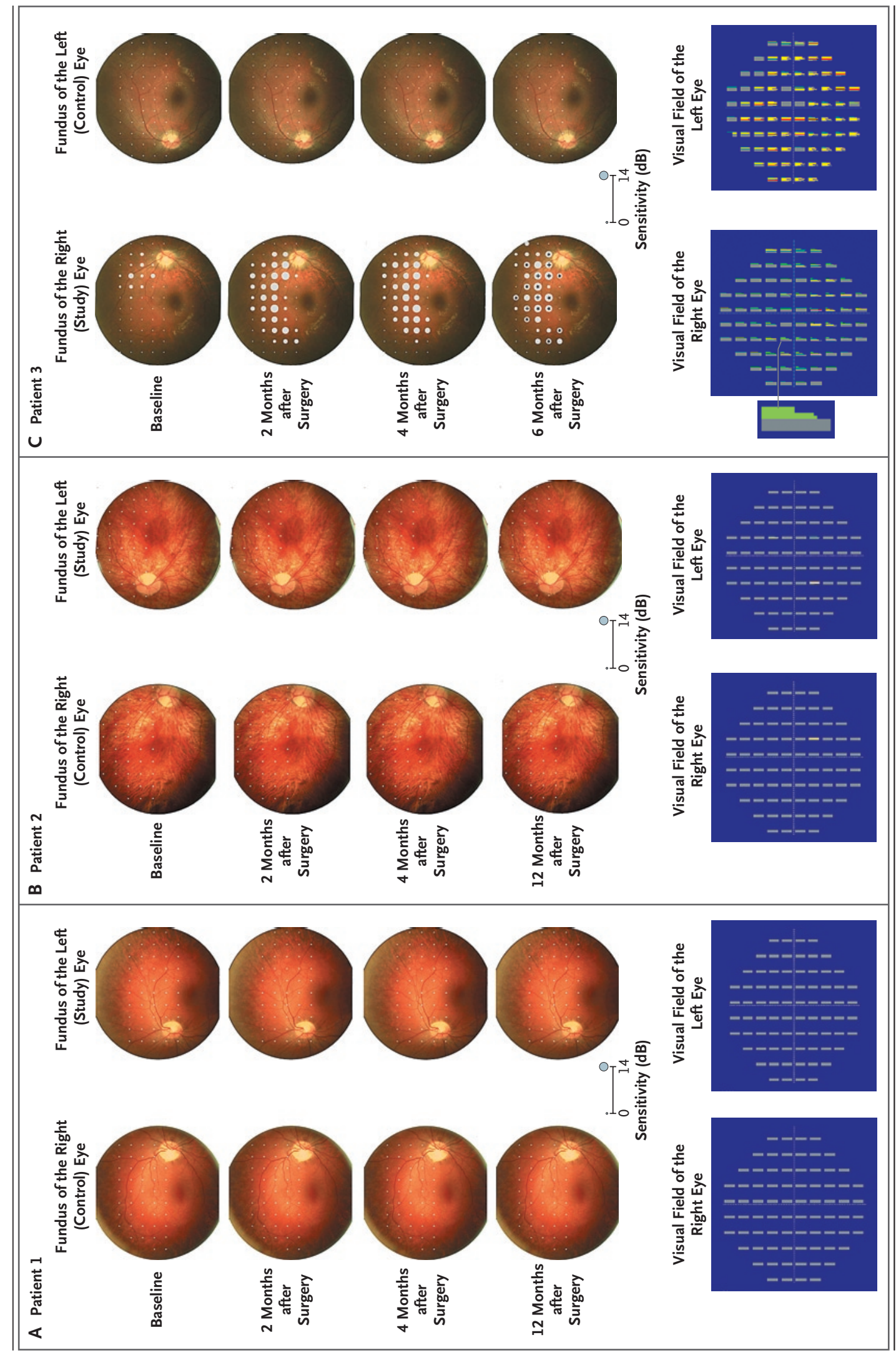


Figure 2 (facing page). Assessment of Visual Function by Microperimetry and Dark-Adapted Perimetry.

The upper portion of each panel shows the microperimetry results for each patient. The size of the circular symbols indicates retinal sensitivity on a scale of 0 to $14 \mathrm{~dB}$. The change in sensitivity in Patient 3 at each tested location from baseline to 6 months' follow-up was evaluated with pointwise linear regression. Of the 55 locations that were tested, 12 (indicated by asterisks) had significant positive slopes $(\mathrm{P}<0.05)$, ranging from 12 to $28 \mathrm{~dB}$ per year. We would expect no more than three points to pass this test by chance alone. A major change in sensitivity of $9 \mathrm{~dB}$ or more (an increase in sensitivity by a factor of 8 ) is indicated by a plus sign. The lower portion of each panel shows the dark-adapted perimetry results for each patient. The analysis provides significance levels for change over time at each individual test location. We made a series of eight measurements during the 6 -month follow-up period. Each measurement is depicted by a bar; the lengths of the bars represent sensitivity, with the long bars showing loss of sensitivity and the short bars showing normal sensitivity. Yellow indicates a decline in sensitivity that is not significant, red indicates a decline that is significant $(P<0.05)$, and green indicates an improvement that is significant $(\mathrm{P}<0.01)$. One example, at the $X / Y$ coordinate $-9,+3$ of the right eye of $\mathrm{Pa}$ tient 3 , is magnified to show the sensitivity measurements going from baseline on the left sequentially through the follow-up assessments on the right. In this example, the long gray bars on the left indicate that the patient was unable to see the light stimulus at maximum intensity. The shorter bars on the right indicate progressive improvement in sensitivity $(\mathrm{P}<0.01)$.

from 77 seconds to 14 seconds for the study eye, and mobility errors decreased from 8 to 0 . Similar results were obtained in a second follow-up test 4 weeks later.

\section{DISCUSSION}

Recombinant adeno-associated virus $2 / 2$ vector efficiently transduces retinal pigment epithelial cells after subretinal delivery in animal models. ${ }^{24}$ We investigated the feasibility of subretinal vector injection in patients with advanced retinal degeneration and found that we could achieve this outcome predictably and without immediate adverse events, using a transvitreal, transretinal approach after pars plana vitrectomy. A relatively degenerate vitreous facilitated detachment and removal of the posterior vitreous cortex, which otherwise might have resisted passage of the fine cannula. We found that we could deliver through a single retinotomy up to $1 \mathrm{ml}$ of vector subreti- nally without causing tears in thinned, degenerate retina. To investigate the effect of RPE65 gene therapy on macular function, we included the macula in the area of vector administration by injection of air into the vitreous cavity when necessary. An important concern was that detachment of the neurosensory retina as a result of subretinal injection could adversely affect vision in the long term, particularly if the detachment involved the macula. We found that the induced retinal detachment resolved spontaneously and fully within a few days after injection, with subsequent recovery of vision to preexisting levels. We did not identify any clinically significant adverse effect of subretinal vector delivery, and the absence of systemic dissemination suggests that any extraocular leakage of vector from the subretinal space was minimal.

To minimize the possibility of intraocular inflammation elicited by vector capsid proteins, we used perioperative systemic immunosuppressive agents. Because of concerns about the possibility of immune responses to the transgene product, we used a tissue-specific promoter and excluded patients with null mutations. We observed no clinically significant intraocular inflammation and detected no immune responses to either adeno-associated virus capsid or RPE65.

We found consistent evidence, on the basis of both microperimetry and dark-adapted perimetry, of improved vision in one patient (Patient 3). The improvement in his visual mobility in low light was also substantially greater than that which would be due to a modest learning effect and was consistent with the improvement in visual function established by means of perimetry. It is not clear whether the improvement in visual responses in the peripheral macula is rod-mediated or cone-mediated. Neither can we be sure that the improvement in visual function is entirely due to enhanced levels of RPE65 in the retina. Evidence for this could be obtained only by biopsy of retinal material, which would be unsafe and unethical. Central macula function and visual acuity did not improve, despite exposure of this region to the vector; this may be due either to amblyopia (i.e., the study eye was amblyopic) or to a requirement for higher levels of RPE65 at the fovea. Visual function improved in only one patient (Patient 3); he had better baseline visual acuity in both the study (amblyopic) eye and the control eye than either of the other 


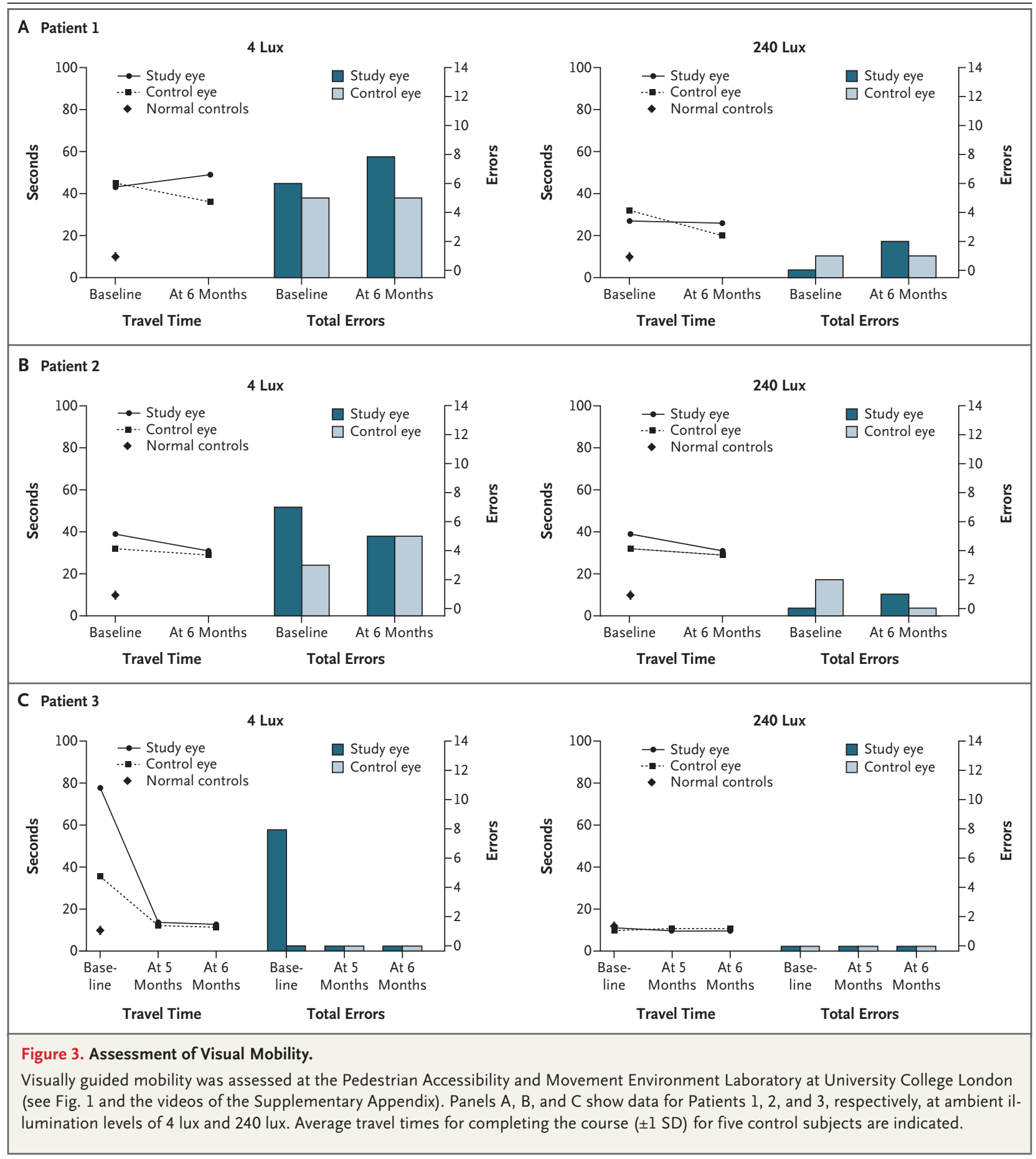

patients. He was not the youngest patient, but he probably had less advanced retinal disease at baseline, which may explain why the improvement in this patient was not observed in the other patients. Whether further retinal degen- eration is delayed in any of the patients will become apparent only after several years.

The results of this study suggest that subretinal administration of recombinant adeno-associated virus vector is not associated with imme- 
diate adverse events in patients with severe retinal dystrophy and that adeno-associated virus-mediated RPE65 gene therapy can lead to modest improvements in visual function, even in patients with advanced degeneration. Our findings provide support for the development of further clinical studies in children with RPE65 deficiency; these children are more likely to benefit than adults.

Supported by grants from the U.K. Department of Health, the British Retinitis Pigmentosa Society, and the Special Trustees of Moorfields Eye Hospital, and by the Sir Jules Thorn Charitable Trust, the Wellcome Trust, the European Union (EVI-Genoret and Clinigene programs), the Medical Research Council, Foundation Fighting Blindness, Fight for Sight, the Ulverscroft Foundation, Fighting Blindness (Ireland), Moorfields Eye Hospital, and Institute of Ophthalmology Biomedical Research Centre for Ophthalmology, University College London.
Drs. Bainbridge and Ali report being the inventors associated with a U.S. patent application assigned to University College London and Targeted Genomics Corporation entitled, "Devices and methods for delivering polynucleotides into retinal cells of the macula and fovea"; Drs. Fitzke and Viswanathan report being two of the inventors of Progressor software, the intellectual property rights for which are owned by University College London and Moorfields Eye Hospital and licensed to Medisoft; Dr. Carter reports being an employee of Targeted Genetics and receiving consulting fees from Sangamo Biosciences. No other potential conflict of interest relevant to this article was reported.

We thank Andrew Dick and the U.K. RPE65 Gene Therapy Data and Safety Monitoring Committee; Alan Bird and the Moorfields Eye Hospital RPE65 Gene Therapy Advisory Committee; Vivien Perry and Moorfields Pharmaceuticals; Graeme Black, for help with genotyping; David Wong, for advice on surgical techniques; and the patients and their families for their effort and commitment.

APPENDIX

The following investigators, who are members of the Moorfields Eye Hospital and University College London Eye Gene Therapy Study Group, participated in this trial: G.W. Aylward, D. Boampong, C. Broderick, P. Buch, C. Childs, Y. Duran, D. Ehlich, S. Falk, M. Feely, T. Fujiyama, F. Ikeji, V. Luong, A. Milliken, R. Maclaren, P. Moradi, F. Mowat, M. Richardson, C. Ripamonti, A.G. Robson, H. Rostron, I. Russell-Eggitt, P. Schlottmann, M. Tschernutter, and N. Wasseem.

\section{REFERENCES}

1. Hanein S, Perrault I, Gerber S, et al. Leber congenital amaurosis: comprehensive survey of the genetic heterogeneity, refinement of the clinical definition, and genotype-phenotype correlations as a strategy for molecular diagnosis. Hum Mutat 2004;23:306-17.

2. Lorenz B, Gyürüs P, Preising M, et al. Early-onset severe rod-cone dystrophy in young children with RPE65 mutations. Invest Ophthalmol Vis Sci 2000;41:273542.

3. Paunescu K, Wabbels B, Preising MN, Lorenz B. Longitudinal and cross-sectional study of patients with early-onset severe retinal dystrophy associated with RPE65 mutations. Graefes Arch Clin Exp Ophthalmol 2005;243:417-26.

4. Thompson DA, Gyürüs P, Fleischer LL et al. Genetics and phenotypes of RPE65 mutations in inherited retinal degeneration. Invest Ophthalmol Vis Sci 2000;41: 4293-9.

5. Gu SM, Thompson DA, Srikumari CR, et al. Mutations in RPE65 cause autosomal recessive childhood-onset severe retinal dystrophy. Nat Genet 1997;17:194-7.

6. Marlhens F, Bareil C, Griffoin JM, et al. Mutations in RPE65 cause Leber's congenital amaurosis. Nat Genet 1997;17:139-41.

7. Morimura H, Fishman GA, Grover SA, Fulton AB, Berson EL, Dryja TP. Mutations in the RPE65 gene in patients with autosomal recessive retinitis pigmentosa or Leber congenital amaurosis. Proc Natl Acad Sci U S A 1998;95:3088-93.

8. Lotery AJ, Namperumalsamy P, Jacobson SG, et al. Mutation analysis of 3 genes in patients with Leber congenital amaurosis. Arch Ophthalmol 2000;118:538-43. 9. Thompson DA, Gal A. Genetic defects in vitamin A metabolism of the retinal pigment epithelium. Dev Ophthalmol 2003; 37:141-54.

10. Redmond TM, Yu S, Lee E, et al. Rpe65 is necessary for production of 11-cis-vitamin $\mathrm{A}$ in the retinal visual cycle. Nat Genet 1998;20:344-51.

11. Mata NL, Moghrabi WN, Lee JS, et al. Rpe65 is a retinyl ester binding protein that presents insoluble substrate to the isomerase in retinal pigment epithelial cells. J Biol Chem 2004;279:635-43.

12. Jin M, Li S, Moghrabi WN, Sun H, Travis GH. Rpe65 is the retinoid isomerase in bovine retinal pigment epithelium. Cell 2005;122:449-59.

13. Moiseyev G, Chen Y, Takahashi Y, Wu $\mathrm{BX}, \mathrm{Ma}$ JX. RPE65 is the isomerohydrolase in the retinoid visual cycle. Proc Natl Acad Sci U S A 2005;102:12413-8.

14. Redmond TM, Poliakov E, Yu S, Tsai JY, Lu Z, Gentleman S. Mutation of key residues of RPE65 abolishes its enzymatic role as isomerohydrolase in the visual cycle. Proc Natl Acad Sci U S A 2005;102: 13658-63.

15. Znoiko SL, Crouch RK, Moiseyev G, Ma JX. Identification of the RPE65 protein in mammalian cone photoreceptors. Invest Ophthalmol Vis Sci 2002;43:1604-9.

16. Wu BX, Moiseyev G, Chen Y, Rohrer $B$, Crouch RK, Ma JX. Identification of RDH10, an all-trans retinol dehydrogenase, in retinal Muller cells. Invest Ophthalmol Vis Sci 2004;45:3857-62.
17. Veske A, Nilsson SE, Narfström K, Gal A. Retinal dystrophy of Swedish Briard/ Briard-beagle dogs is due to a 4-bp deletion in RPE65. Genomics 1999;57:57-61. 18. Acland GM, Aguirre GD, Ray J, et al. Gene therapy restores vision in a canine model of childhood blindness. Nat Genet 2001;28:92-5.

19. Narfström K, Katz ML, Bragadottir R, et al. Functional and structural recovery of the retina after gene therapy in the RPE65 null mutation dog. Invest Ophthalmol Vis Sci 2003;44:1663-72.

20. Narfström K, Katz ML, Ford M, Redmond TM, Rakoczy E, Bragadottir R. In vivo gene therapy in young and adult RPE65-1- dogs produces long-term visual improvement. J Hered 2003;94:31-7.

21. Le Meur G, Stieger K, Smith AJ, et al. Restoration of vision in RPE65-deficient Briard dogs using an AAV serotype 4 vector that specifically targets the retinal pigmented epithelium. Gene Ther 2007;14: 292-303.

22. Aguirre GK, Komáromy AM, Cideciyan AV, et al. Canine and human visual cortex intact and responsive despite early retinal blindness from RPE65 mutation. PLoS Med 2007;4(6):e230.

23. Gao GP, Qu G, Faust LZ, et al. Hightiter adeno-associated viral vectors from a Rep/Cap cell line and hybrid shuttle virus. Hum Gene Ther 1998;9:2353-62.

24. Auricchio A, Rolling F. Adeno-associated viral vectors for retinal gene transfer and treatment of retinal diseases. Curr Gene Ther 2005;5:339-48.

Copyright (c) 2008 Massachusetts Medical Society. 